\title{
Accessibility of nanotube inner channels investigated by krypton adsorption
}

\author{
T. N. H. Le, ${ }^{1}$ F. Valsaque, ${ }^{1}$ M. Mercy, ${ }^{2}$ J. Ghanbaja, ${ }^{3}$ A. Kohler, ${ }^{3}$ E. McRae ${ }^{1}$ \\ ${ }^{1}$ LCSM, Nancy-Université, CNRS, B.P. 249, 54506 Vandøuvre-lès-Nancy, France \\ ${ }^{2}$ LCAH, Nancy-Université, CNRS, B.P. 249, 54506 Vandœuvre-lès-Nancy, France \\ ${ }^{3}$ SCMET, Nancy-Université, CNRS, B.P. 249, 54506 Vandouvre-lès-Nancy, France \\ Ha.le@lcsm.uhp-nancy.fr
}

\begin{abstract}
Introduction
Because of their remarkable properties, single wall carbon nanotubes (SWCNTs) have been considered for many potential applications in nanoelectronics, as scanning probes, as gas sensors or for field emission displays. ${ }^{1-3}$ Such bulk applications demand large quantities of this material with high purity and homogeneous properties. A high pressure carbon monoxide disproportionation (HiPco) process is one of the several approaches currently attempting to meet these demands. This technique has been shown to produce nanotubes of good purity. ${ }^{4}$ However purification procedures involving acid treatment and/or air-oxidation are still indispensable to remove impurities. But it is well known that purification introduces some detrimental effects such as damaging the tubes or modifying their surfaces through addition of functional groups. ${ }^{5}$ Consequently, characterization of purified samples is necessary. In this work, we report the characterization of HiPco SWCNTs by krypton $\mathrm{Kr}$ adsorption at $77.3 \mathrm{~K}$ in order to analyze the effects of purification on the bundle surface.
\end{abstract}

\section{Experimental section}

Two SWCNT samples (raw and purified) prepared by the HiPco process, were purchased from Carbon Nanotechnologies Inc. at Huston, Texas.

In this study, energy dispersive X-ray spectroscopy (EDX) was utilized for chemical analysis with a Hitachi S4800 field emission gun scanning electron microscopy (FEG-SEM). Transmission electron microscopy (TEM) using a Philips CM 20 apparatus with an unsaturated $\mathrm{LaB}_{6}$ cathode was done to analyse the structure of the nanotube samples. For the TEM observations, raw and purified SWCNTs were dispersed in ethanol using a sonication bath for a few minutes and deposited on a holey copper grid.

The experimental adsorption setup is described elsewhere. ${ }^{6-8}$ The SWCNT samples were maintained at $77.3 \mathrm{~K}$ during experiments. Pressure determinations were performed in an apparatus equipped with McLeod gauges allowing measurements between $10^{-4}$ and $1300 \mathrm{~Pa}$. Thermal transpiration was taken into account by using the semi-empirical equation of Takaishi and Sensui. ${ }^{9}$ Krypton of $99.99 \%$ purity was supplied by "Air Liquide". The gas was purified before each experiment by pumping on the phase condensed at $77.3 \mathrm{~K}$ inside the measurement cell. The sample mass typically used for each measurement was around $10 \mathrm{mg}$. Prior to measurement, the samples were degassed by heating under vacuum at $873 \mathrm{~K}$. Adsorption isotherms of $\mathrm{Kr}$ at $77.3 \mathrm{~K}$ were then measured from the very first stage of adsorption $\left(\mathrm{P} / \mathrm{P}_{0} \sim 10^{-6}\right)$ to the adsorbed saturated vapour pressure $\mathrm{P}_{0}\left(\mathrm{P}_{0}=228 \mathrm{~Pa}\right)$.

\section{Results and discussion}

\section{Chemical characterization}

The mass proportion of residual iron catalyst for HiPco SWCNTs has been evaluated elsewhere by thermogravimetric analysis: Du et al reported a Fe content of approximately $24 \mathrm{wt} . \%,{ }^{10}$, Cinke et $a l^{11}$ a value of $22 \mathrm{wt} . \%$, while Matranga and Bockgrath. ${ }^{12}$ gave 18 wt.\%. Since the impurity content varies from sample to sample, a systematic chemical characterization is necessary. 
In this study, we have estimated the chemical composition by EDX. This technique also allows us to detect the presence of elements other than iron $(\mathrm{Cl}, \mathrm{O}, \mathrm{C} \ldots)$. In order to obtain representative results of the whole sample, the electron beam was focused on a quite large surface area (1 $\mathrm{mm}^{2}$ ) with a detection depth of $\sim 1 \mu \mathrm{m}$. The analyses were repeated three times for each sample and each gave similar results. The sample compositions of two analyses are reported in Table 1.

EDX revealed an increase in carbon content from 70.3 to 87.0 wt. \%, accompanied by a decrease of iron and oxygen amounts. Nevertheless, the purification could not completely remove the residual catalyst from the

Table 1. Sample composition in wt. \% determined by EDX.

\begin{tabular}{|l|c|c|}
\hline & Raw (wt. \%) & Purified (wt. \%) \\
\hline $\mathrm{C}$ & $70.3 \pm 0.3$ & $87.0 \pm 0.5$ \\
\hline $\mathrm{O}$ & $14.2 \pm 0.2$ & $5.7 \pm 0.2$ \\
\hline $\mathrm{Cl}$ & $0.1 \pm 0.1$ & $3.5 \pm 0.5$ \\
\hline $\mathrm{Fe}$ & $15.0 \pm 0.5$ & $3.7 \pm 0.6$ \\
\hline Others & $0.4 \pm 0.1$ & $0.1 \pm 0.1$ \\
\hline
\end{tabular}

sample. Furthermore, the purification has introduced a chlorous contamination, perhaps in the form of $\mathrm{FeCl}_{3}$.

\section{TEM observations}

From TEM analysis, as shown in Fig. 1, we do not observe any graphitic particles and only a very small amount of amorphous carbon. The carbon mass proportion is thus representative of the SWCNT proportion in the sample. Residual catalysts were found on the raw bundle surface (left image) but most of them were removed after the purification treatment (right image). This is in agreement with the EDX analysis (decrease of iron content).
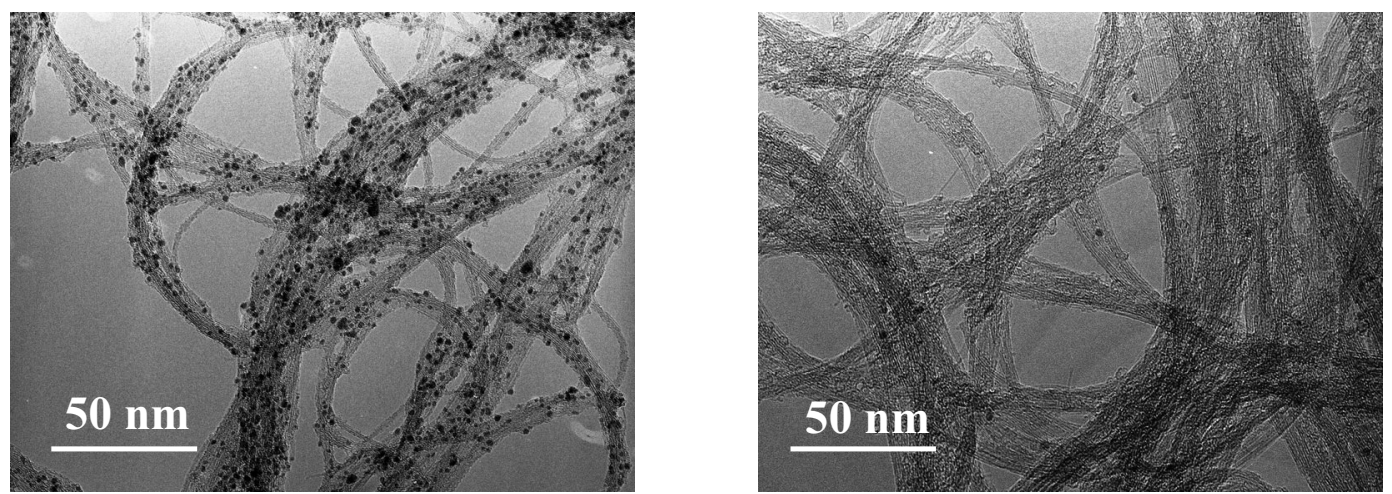

Fig. 1. TEM photos of (left) raw sample and (right) purified sample.

\section{Gas adsorption studies}

Adsorption isotherms (i. e. the amount of $\mathrm{Kr}$ adsorbed on SWCNTs versus the equilibrium $\mathrm{Kr}$ pressure) are presented in Fig. 2. Each adsorption isotherm exhibits two inclined steps, which is consistent with previous findings on gas adsorption on SWCNTs. ${ }^{6,7,13-16}$

In the case of formation of successive condensed monolayer on the same adsorption sites $(\mathrm{Kr}$ adsorption on exfoliated graphite for example ${ }^{17-19}$ ) the step heights are identical because each adsorbed layer corresponds to condensation of the same adsorbate amount. On the other hand, in our case, we clearly observe that the heights of the first and second steps are not equal. This indicates that the two steps are characteristic of two different kinds of adsorption sites. 
Fig. 2. Krypton adsorption isotherms at $77 \mathrm{~K}$ (normalised to the sample weight). The raw and purified samples correspond to the lower and upper curves, respectively. The dashed curve is obtained from $\mathrm{C}_{1} \mathrm{I}_{\text {raw }}(\mathrm{P})+\mathrm{C}_{2}$, where $\mathrm{I}_{\text {raw }}(\mathrm{P})$ is the adsorbed amount on the raw sample at the pressure $\mathrm{P}, \mathrm{C}_{1}=87.0 / 70.3$ and $\mathrm{C}_{2}=3.3 \mathrm{mmol} / \mathrm{g}$.

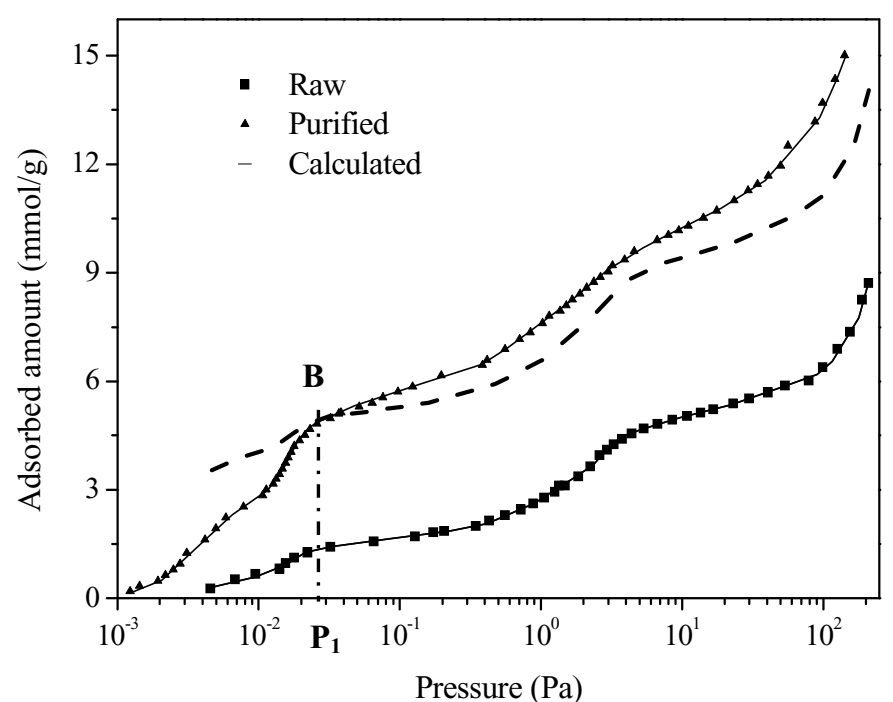

According to literature, ${ }^{6,7,13-16}$ the existence of different adsorption sites on SWCNTs is the result of bundle formation. The different potential adsorption sites are: the interstitial channels, the inner channels (if tubes are open), the grooves and the external nanotube walls (Fig. 3). Among these, the most energetic sites are the grooves and the inner channels. Their adsorption energies are similar and correspond to the first step in the adsorption isotherms. The second step corresponds to the less attractive sites which are the external walls. Concerning the interstitial channels, even if $\mathrm{Kr}$ atoms are small enough to enter, the associated adsorption energy is so low, that their adsorption can be neglected. ${ }^{16}$

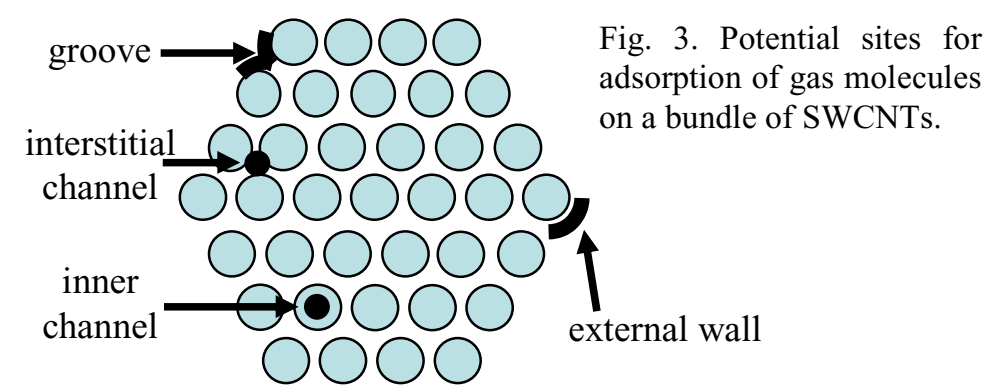

The BET method gives a specific surface area (SSA) of $(420 \pm 20) \mathrm{m}^{2} / \mathrm{g}$ for the raw sample. This is in good agreement with the work of Krungleviciute et al., ${ }^{20}$ in which surface area measurements were determined via four different adsorbates: neon, argon, xenon, and methane. In their study, estimated SSA values range from 450 to $468 \mathrm{~m}^{2} / \mathrm{g}$ for the same sample. In our case, the SSA is multiplied by a factor of about 2 after purification: $(858 \pm 30) \mathrm{m}^{2} / \mathrm{g}$. If we suppose that there are no carbon impurities, i.e., SWCNTs are the only carbon species in the samples, then the increase in SWCNT mass proportion following purification can only give rise to an enhancement of $\mathrm{C}_{1}=87 / 70.3$ in SSA (see table 1). It is thus clear that there is another reason behind the increase observed on Fig. 2. In order to identify this, a calculated isotherm (the dashed curve in Fig. 2) has been added. This curve is obtained by: $I_{\text {calculated }}(P)=C_{1} I_{\text {raw }}(P)+C_{2}$, where $C_{2}$ is an constant chosen so that $\mathrm{I}_{\text {calculated }}\left(\mathrm{P}=\mathrm{P}_{1}\right)=\mathrm{I}_{\text {purified }}\left(\mathrm{P}=\mathrm{P}_{1}\right)$ (where $\mathrm{P}_{1}$ is the pressure at the end of the first step, i.e., the point $\mathrm{B})$. We see that there is only a small differences between $\mathrm{I}_{\text {calculated }}(\mathrm{P})$ and $\mathrm{I}_{\text {purified }}(\mathrm{P})$ for $\mathrm{P}>\mathrm{P}_{1}$. In particular, the second step heights (corresponding to adsorption on external walls) are similar. We conclude that the purification does not significantly change the external walls of SWCNT bundles. On the other hand, we note that the height of the first step for the purified sample is much greater than that of the raw one. If we consider that purification does 
not affect the groove sites (as for the external walls), then this increase from raw to purified samples can only be attributed to adsorption in the inner channels.

It has been well established that many purification treatments oxidise the nanotube surface and introduce various functional groups, usually located at the tube ends and on wall defects sites. These groups may block gases from entering the inner channels. The heat-treatment prior to adsorption measurements in our case may thus have been sufficient to decompose or remove these groups or promote their steric rearrangement and thus render the inner channels accessible. 12,21

\section{Conclusions}

Physisorption has shown itself to be a very useful characterization technique for investigating the surface properties of carbon nanotubes. Adsorption results show that increased SSA is mainly due to the opening of the SWCNTs so that adsorption can occur in their inner channels. A rough calculation allows estimating that most of the tubes have been opened. The results further illustrate that the, increased SWCNT mass proportion plays a smaller role in the isotherm evolution upon purification. Future work will aim at better understanding details of the adsorption process on HiPco tubes for which the sample morphology is often intrinsically complex with bundles comprising tubes of many diameters.

\section{Acknowledgement}

The authors thank P. Launois, V. Heresanu and C. Bussy for supplying the samples and for many fruitful discussions.

1. M. Meyyappan, Carbon Nanotubes: Science and Applications. CRC Press (2003).

2. A. Loiseau, P. Launois, P. Petit, S. Roche and J.-P. Salvetat, Understanding Carbon Nanotubes, Springer (2006).

3. M.S. Dresselhaus, G. Dresselhaus and P. Avouris, Carbon Nanotubes Synthesis, Structure, Properties, and Applications. Springer-Verlag (2001).

4. P. Nikolaev, M.J. Bronikowski, R.K. Bradley, F. Rohmund, D.T. Colbert, K.A. Smith and R.E. Smalley, Chem. Phys. Lett. 313, 91 (1999).

5. S. Arepalli, P. Nikolaev, O. Gorelik, V.G. Hadjiev, W. Holmes, B. Files and L. Yowell, Carbon 42, 1783 (2004).

6. M. Muris, N. Dupont-Pavlovsky, M. Bienfait and P. Zeppenfeld, Surface Science 492, 67 (2001).

7. E. McRae, M.R. Babaa, F. Valsaque and K. Masenelli-Varlot, Recent research developments in surface science 1, 51 (2004).

8. M.R. Babaa, N. Dupont-Pavlovsky, E. McRae and K. Masenelli-Varlot, Carbon 42, 1549 (2004).

9. T. Takaishi, S. Sensui, Trans. Faraday. Soc. ,59, 2503 (1963).

10. W.F. Du, L. Wilson, J. Ripmeester, R. Dutrisac, B. Simard and S. Denommee, Nano Lett. 2,343 (2002).

11. M. Cinke, J. Li, C.W. Bauschlicher, A. Ricca and M. Meyyappan, Chem. Phys. Lett. 376, 761 (2003).

12. C. Matranga and B. Bockrath, J. Phys. Chem. B 109, 4853 (2005).

13. S. Talapatra, A.Z. Zambano, S.E. Weber and A.D. Migone, Physical Review Letters 85, 138 (2000).

14. D.S. Rawat, L. Heroux, V. Krungleviciute and A.D. Migone, Langmuir 22, 234 (2006).

15. M.R. Babaa, I. Stepanek, K. Masenelli-Varlot, N. Dupont-Pavlovsky, E. McRae and P. Bernier, Surface Science 531, 86 (2003).

16. M. Arab, F. Picaud, C. Ramseyer, M.R. Babaa, F. Valsaque and E. McRae, J. Chem. Phys. 126, 054709 (2007).

17. A. Thomy, X. Duval and J. Regnier, Surface Science Reports 1, 1 (1981).

18. A. Thomy and X. Duval, Surface Science 299/300, 415 (1994).

19. A. Thomy and X. Duval, J. Chim. Phys. 67, 1966 (1969).

20. V. Krungleviciute, L. Heroux, S. Talapatra and A.D. Migone, Nano Lett. 4, 1133 (2004).

21. A. Kuznetsova, D.B. Mawhinney, V. Naumenko, J.T. Yates Jr, J. Liu and R.E. Smalley, Chem. Phys. Lett. 321, $292(2000)$. 